\title{
Age-dependent effects of brain stimulation on network centrality
}

Antonenko, Daria ; Nierhaus, Till; Meinzer, Marcus; Prehn, Kristin; Thielscher, Axel; Ittermann, Bernd; Flöel, Agnes

\section{Published in:}

Neurolmage

Link to article, DOI:

10.1016/j.neuroimage.2018.04.038

Publication date:

2018

Document Version

Peer reviewed version

Link back to DTU Orbit

Citation (APA):

Antonenko, D., Nierhaus, T., Meinzer, M., Prehn, K., Thielscher, A., Ittermann, B., \& Flöel, A. (2018). Agedependent effects of brain stimulation on network centrality. Neurolmage, 176, 71-82.

https://doi.org/10.1016/j.neuroimage.2018.04.038

\section{General rights}

Copyright and moral rights for the publications made accessible in the public portal are retained by the authors and/or other copyright owners and it is a condition of accessing publications that users recognise and abide by the legal requirements associated with these rights.

- Users may download and print one copy of any publication from the public portal for the purpose of private study or research.

- You may not further distribute the material or use it for any profit-making activity or commercial gain

- You may freely distribute the URL identifying the publication in the public portal

If you believe that this document breaches copyright please contact us providing details, and we will remove access to the work immediately and investigate your claim 


\section{Accepted Manuscript}

Age-dependent effects of brain stimulation on network centrality

Daria Antonenko, Till Nierhaus, Marcus Meinzer, Kristin Prehn, Axel Thielscher, Bernd Ittermann, Agnes Flöel

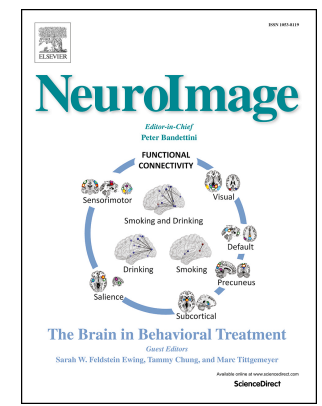

PII:

S1053-8119(18)30348-3

DOI:

10.1016/j.neuroimage.2018.04.038

Reference: $\quad$ YNIMG 14885

To appear in: Neurolmage

Received Date: 22 November 2017

Revised Date: 13 April 2018

Accepted Date: 17 April 2018

Please cite this article as: Antonenko, D., Nierhaus, T., Meinzer, M., Prehn, K., Thielscher, A., Ittermann, B., Flöel, A., Age-dependent effects of brain stimulation on network centrality, Neurolmage (2018), doi: 10.1016/j.neuroimage.2018.04.038.

This is a PDF file of an unedited manuscript that has been accepted for publication. As a service to our customers we are providing this early version of the manuscript. The manuscript will undergo copyediting, typesetting, and review of the resulting proof before it is published in its final form. Please note that during the production process errors may be discovered which could affect the content, and all legal disclaimers that apply to the journal pertain. 


\section{Age-dependent effects of brain stimulation on network}

\section{centrality}

Daria Antonenko ${ }^{\mathrm{a}, \mathrm{b}}$, Till Nierhaus ${ }^{\mathrm{c}, \mathrm{d}}$, Marcus Meinzer ${ }^{\mathrm{e}}$, Kristin Prehn ${ }^{\mathrm{a}}$, Axel Thielscher ${ }^{\mathrm{f}, \mathrm{g}}$, Bernd Ittermann ${ }^{\mathrm{h}}$, Agnes Flöel $\mathrm{a}^{\mathrm{a}, \mathrm{b}}$

${ }^{\text {a} C h a r i t e ́ ~-~ U n i v e r s i t a ̈ t s m e d i z i n ~ B e r l i n, ~ c o r p o r a t e ~ m e m b e r ~ o f ~ F r e i e ~ U n i v e r s i t a ̈ t ~ B e r l i n, ~ H u m b o l d t-~}$ Universität zu Berlin, and Berlin Institute of Health, Department of Neurology, NeuroCure Clinical Research Center, Charitéplatz 1, 10117 Berlin, Germany; ${ }^{\mathrm{b}}$ Department of Neurology, University of Greifswald, 17475 Greifswald, Germany; 'Max-Planck-Institute for Human Cognitive and Brain Sciences, Department of Neurology, 04303 Leipzig, Germany, ${ }^{\mathrm{d}}$ Center for Cognitive Neuroscience Berlin (CCNB), Department of Education and Psychology, Freie Universität Berlin, Germany; ${ }^{\mathrm{e}}$ The University of Queensland, Centre for Clinical Research, Brisbane Queensland, 4029, Australia; fDanish Research Centre for Magnetic Resonance, Centre for Functional and Diagnostic Imaging and

Research, Copenhagen University Hospital Hvidovre, Denmark; ${ }^{9}$ Center for Magnetic Resonance, Department of Electrical Engineering, Technical University of Denmark, Kgs Lyngby, Denmark; ${ }^{\text {h}}$ Physikalisch-Technische Bundesanstalt (PTB), Abbestr. 2, 10587 Berlin, Germany

Correspondence should be addressed to Dr. Daria Antonenko, daria.antonenko@unigrefiswald.de; or Prof. Dr. Agnes Flöel, Department of Neurology, Universitätsmedizin Greifswald, Ferdinand-Sauerbruch-Straße, 17475 Greifswald, +49 383486 6815, agnes.floeel@uni-greifswald.de 


\section{Abstract}

Functional magnetic resonance imaging (fMRI) studies have suggested that advanced age may mediate the effects of transcranial direct current stimulation (tDCS) on brain function. However, studies directly comparing neural tDCS effects between young and older adults are scarce and limited to task-related imaging paradigms. Resting-state (rs-) fMRI, that is independent of age-related differences in performance, is well suited to investigate ageassociated differential neural tDCS effects. Three "online" tDCS conditions (anodal, cathodal, sham) were compared in a cross-over, within-subject design, in 30 young and 30 older adults. Active stimulation targeted the left sensorimotor network (active electrode over left sensorimotor cortex with right supraorbital reference electrode). A graph-based rs-fMRI data analysis approach (eigenvector centrality mapping) and complementary seed-based analyses characterized neural tDCS effects. An interaction between anodal tDCS and age group was observed. Specifically, centrality in bilateral paracentral and posterior regions (precuneus, superior parietal cortex) was increased in young, but decreased in older adults. Seed-based analyses revealed that these opposing patterns of tDCS-induced centrality modulation were explained from differential effects of tDCS on functional coupling of the stimulated left paracentral lobule. Cathodal tDCS did not show significant effects. Our study provides first evidence for differential tDCS effects on neural network organization in young and older adults. Anodal stimulation mainly affected coupling of sensorimotor with ventromedial prefrontal areas in young and decoupling with posteromedial areas in older adults.

\section{Keywords}

Aging; eigenvector centrality mapping; graph analysis; resting-state functional connectivity; transcranial direct current stimulation 


\section{Introduction}

Non-invasive brain stimulation techniques may constitute promising approaches to investigate the relationship between brain structure and function and to develop cognitive enhancement strategies (Fertonani and Miniussi, 2016; Kuo and Nitsche, 2012; Nitsche et al., 2015). Importantly, such techniques have been discussed as a potential intervention to counteract age-related cognitive decline and reduced neural efficacy (Mameli et al., 2014; Perceval et al., 2016).

Here, transcranial direct current stimulation (tDCS) has proven to be the most feasible method, because of its relative low costs, few contraindications and adverse effects and its simplicity for concurrent application during task execution (Polania et al., 2018; Woods et al., 2016). When applied concurrent to cognitive training over multiple days, tDCS has been found to induce sustained performance improvement of trained and untrained functions (Antonenko et al., 2018; Berryhill, 2017; Kuo and Nitsche, 2012). In addition to its additive effect on training interventions, there is a large number of studies using single session tDCS to establish brain-behavior relationships and study underlying neural effects (Perceval et al., 2016; Polania et al., 2018). At the neural level, acute tDCS effects are modulation of cortical excitability by de- or hyperpolarizing resting membrane potentials (Nitsche and Paulus, 2000; Polania et al., 2018). Applied over a longer time interval, tDCS effects have been suggested to include synaptic mechanisms leading to long-term potentiation- (LTP) and depression-like plasticity (Monte-Silva et al., 2013; Nitsche and Paulus, 2001; Stagg and Nitsche, 2011). In particular, LTP-like effects induced by anodal tDCS can extend to interconnected brain areas, exerting network-based neurophysiological modulation that is evident during current application and can last up to an hour after the stimulation ends (Polania et al., 2018; Sehm et al., 2012; Stagg and Nitsche, 2011). Importantly, magnitude and direction of neurophysiological and behavioral effects of tDCS may not only depend on external methodological factors (e.g., stimulation parameters including electrode montage, current strength and stimulation duration), but also on inter-individual (e.g., genotype, baseline 
performance and education) and intra-individual factors (e.g., brain state) (Krause and Cohen Kadosh, 2014; Polania et al., 2018; Stephens et al., 2017).

In order to investigate effects on neural processing, brain stimulation can be combined with brain imaging techniques (for an overview, see Bergmann et al., 2016; Siebner et al., 2009). These include electroencephalography, magnetoencephalography, functional near-infrared spectroscopy and functional magnetic resonance imaging (fMRI) - each having its advantages, limits and technical challenges regarding the temporal and spatial resolution with which they can map certain neural patterns (Bolognini and Miniussi, 2016; Jones et al., 2015; Miniussi et al., 2012). The main advantage of fMRI over other techniques is that it can provide whole-brain information of stimulation effects on both local activity and large scale functional network with high spatial and sufficient temporal resolution (Antal et al., 2011; Johnstone et al., 2016; Siebner et al., 2009; Woods et al., 2016).

Using fMRI assessment, several studies have described tDCS-induced modulations of functional brain networks comprising effects in areas proximal and distant to the stimulation electrodes using both task-dependent and resting-state fMRI assessments in young adults (Bachtiar et al., 2015; Lindenberg et al., 2016; Martin et al., 2017; Meinzer et al., 2012; Sehm et al., 2012). Investigations of tDCS-induced functional modulation in older adults are scarce but essential, given differential effects in young versus older cohorts due to prominent agerelated reorganization (cf. Perceval et al., 2016; Summers et al., 2016). Studies with older participants, in fact, allow to anticipate age-specific patterns of tDCS-induced modulations (Antonenko et al., 2017; Lindenberg et al., 2013). For instance, in a recent study we found that functional connectivity in the sensorimotor network is reduced due to anodal tDCS in older participants (Antonenko et al., 2017), as compared to an increase previously reported in young adults (Bachtiar et al., 2015).

Only one study to date included both older and young adults and compared the effects of tDCS on underlying functional connectivity during a semantic word generation task (Martin et al., 2017). The authors found performance improvements during tDCS administered to the 
left sensorimotor cortex and overlapping functional network modulations as assessed by independent component analysis during the task in both age groups. Only older adults showed increased lateralization of language related networks during anodal tDCS. Thus, tDCS resulted in partially different effects in both age groups. Effects on blood-oxygenated level-dependent (BOLD) task $\mathrm{fMRI}$ were related to the experimental paradigm, stimulus input and behavioral output. Here, task-independent resting-state BOLD fMRI not only offers a promising approach avoiding confounds related to the interaction of brain activity during task performance, but may also facilitate the understanding of complex network organization and stimulation-induced neuromodulation in aging (Ferreira and Busatto, 2013; Fox and Raichle, 2007).

The present study compared brain stimulation response in groups of older and young participants during task-free resting-state fMRI. Specifically, we administered anodal, cathodal and sham tDCS over the left sensorimotor cortex (SM1) with a right supraorbital (SO) reference electrode using a within-subjects design. Resting-state fMRI was acquired in all tDCS conditions during stimulation. For connectivity analysis, we chose the established graph theory-based eigenvector centrality mapping (ECM) approach, because it is purely data-driven and allows characterization of whole-brain functional connectivity without requiring a priori assumptions about anatomical or functional network organization or regions-of-interest specification (Bonacich, 2007; Lohmann et al., 2010; Nierhaus et al., 2012; Zuo et al., 2012). In addition, this approach has successfully been used to map tDCSinduced modulations of resting-state functional connectivity in several previous studies in young or older adults (Lindenberg et al., 2013; Lindenberg et al., 2016; Meinzer et al., 2012; Meinzer et al., 2015; Sehm et al., 2012).

Based on substantial evidence of age-related brain network reorganization (Ferreira and Busatto, 2013; Geerligs et al., 2015; Sala-Llonch et al., 2015) and previous fMRI studies that pointed towards age group-specific patterns of neural tDCS effects on brain activity and connectivity (Lindenberg et al., 2013; Martin et al., 2017; Meinzer et al., 2012), we aimed to 
investigate the interaction between stimulation condition and age group. Thus, we focused on differences in whole-brain spatial distribution of tDCS-induced neural effects with the aim to explore which regions showed modulatory patterns in different directions in older and young adults. Based on available preliminary data described above, we expected differential large-scale network modulations in both age groups with inter-regional connectivity of areas surrounding the targeted sensorimotor cortex to be increased during anodal stimulation in young adults (Bachtiar et al., 2015; Lindenberg et al., 2016) and to be decreased in older adults (Antonenko et al., 2017).

\section{Materials and Methods}

\subsection{Participants and study design}

The study sample comprised sixty participants, 30 older ( $16 \mathrm{f}$, mean/SD age: 63/7, mean/SD education: 16/3 years) and 30 young adults (16 f, mean/SD age: 24/4, mean/SD education: $16 / 3$ years). All were native German speakers and had no history of neurological or psychiatric disorders. Intake of medication affecting the central nervous-system was treated as an exclusion criterion. Smoking was not an exclusion criterion, but the proportion of smokers in the study sample was low ( $n=2$ in each age group). Older participants were administered the CERAD-Plus test battery (Consortium to Establish a Registry for Alzheimer's Disease; www.memoryclinic.ch) to assure normal cognitive functioning. The study was approved by the Ethics Committee of the Charité Universitätsmedizin Berlin and was conducted in accordance with the Declaration of Helsinki. Written informed consent was obtained from all participants prior to study inclusion.

All older and young adults participated in three MRI sessions, separated by 1 week. Before scanning, tDCS electrodes were attached to the head targeting the sensorimotor network with the "active" electrode over the left sensorimotor cortex (SM1) with a right supraorbital (SO) reference electrode (SM1-SO montage). Electrical current application started concurrent to the beginning of resting-state fMRI acquisition. Two consecutive echoplanar imaging sequences (of 6 min each, see below) were acquired with a 3-min break in-between. 


\section{ACCEPTED MANUSCRIPT}

The order of anodal, cathodal and sham stimulation conditions was counterbalanced over the MRI sessions. For the present study we acquired data of young participants whereas restingstate fMRI data of older participants (selecting an education- and gender-matched subsample of an equal number of participants) was used from a previous study of our group (Antonenko et al., 2017).

\section{2. tDCS}

Setup of intra-scanner tDCS was identical to our previous studies (Antonenko et al., 2017; Lindenberg et al., 2013; Meinzer et al., 2014). A battery-driven MRI-compatible stimulation device (NeuroConn DC-Stimulator Plus, NeuroCare Group, Munich, Germany) positioned outside the scanner room was used to deliver electrical current to the scalp. Two filter boxes were placed between stimulator and electrodes, one inside and one outside the scanner room to absorb radio frequency noise, and $5 \mathrm{kOhm}$ resistors were included in both electrode cables. Electrode montage corresponded to the classical montage in order to target the sensorimotor system, i.e., the "active" electrode (anode in anodal stimulation condition, cathode in cathodal stimulation condition) was centered over the left SM1 with the reference electrode over the right supraorbital region. Electrodes were inserted into saline-soaked sponges ( $5 \times 7 \mathrm{~cm}^{2}$ for active and $10 \times 10 \mathrm{~cm}^{2}$ for reference) and placed according to the 1020 EEG system (C3 for active and Fp2 for reference). Current was delivered with an intensity of $1 \mathrm{~mA}$ for $15 \mathrm{~min}$ with $10 \mathrm{~s}$ fade in/out intervals (beginning with the resting-state fMRI sequence) in active stimulation conditions and for $30 \mathrm{~s}$ in sham stimulation condition.

Before and after each stimulation condition, the positive and negative affect schedule (PANAS) was administered where participants had to rate their current mood on 10 positive and 10 negative items (from 1 to 5) (Watson et al., 1988). After completion of the last MRI session, participants had to retrospectively report the occurrence of adverse effects during stimulation in a questionnaire (adapted from Poreisz et al., 2007).

\subsubsection{Computation of the electric field distributions}


The software SimNIBS 2.0 was used to calculate the electric field induced by tDCS based on the finite element method (FEM) and individualized head models derived from the structural MR images (http://simnibs.org) (Thielscher et al., 2015; Windhoff et al., 2013). Please see Supplementary Methods for a description of the simulation parameters and the details of the simulation procedures. After the field calculations, the middle layer of the cortical sheet was estimated from the pial and white matter surfaces provided by FreeSurfer. The electric field strength (i.e., the vector norm of the electric field E) and the field component that is orthogonal to the cortical sheet were then extracted at the middle layer (see Bungert et al., 2017 for more details on these procedures). The individual results were transformed to the average surface 'fsaverage' provided by Freesurfer, and group averages and standard deviations of the electric field strength and the normal component were calculated for both age groups.

\subsection{MRI data acquisition}

MRI scans were acquired on a 3 tesla Siemens Verio scanner using a 32-channel head coil (with electrodes already attached). High-resolution anatomical images were acquired using a three-dimensional T1-weighted magnetization-prepared rapid acquisition gradient echo (MPRAGE, repetition time: $2300 \mathrm{~ms}$, echo time: $3.03 \mathrm{~ms}$; inversion time: $900 \mathrm{~ms}$; flip angle: $9^{\circ} ; 256 \times 256 \times 192$ matrix; $1 \mathrm{~mm}^{3}$ isotropic voxel). For resting-state fMRI two consecutive echoplanar imaging sequences $\left(3 \times 3 \times 4 \mathrm{~mm}^{3}\right.$, TR: $2300 \mathrm{~ms}$, TE: $30 \mathrm{~ms}$; flip angle: $90^{\circ} ; 35$ slices; no gap; interleaved acquisition; field of view: $192 \times 192 \mathrm{~mm}^{2}$; matrix: $64 \times 64 ; 150$ volumes), with a 3-min interval in-between, were acquired. Subjects were instructed to keep their eyes closed, to try not to fall asleep, and to think of nothing particular. None of the participants had fallen asleep during the stimulation interval, as verified by interviewing the participants after each scanning session.

\subsection{MRI data analyses}

\subsubsection{MRI Preprocessing}


Imaging data were processed using software tools implemented in MATLAB (The MathWorks Inc., Natick, MA; www.mathworks.com). Structural images that were acquired during the sham stimulation condition were segmented into grey matter, white matter and cerebral spinal fluid. A study-specific anatomical template was created using DARTEL to optimize inter-individual realignment (Ashburner, 2007). Preprocessing of functional images was performed using Statistical Parametric Mapping, SPM12 (Wellcome Department of Imaging Neuroscience, London, UK; www.fil.ion.ucl.ac.uk/spm/) and included slice timing correction, head motion correction, co-registration to individual structural T1 images, spatial normalization, spatial smoothing (full width half maximum $6 \mathrm{~mm}$ isotropic Gaussian kernel) and temporal filtering (high-pass at $100 \mathrm{~s}=0.01 \mathrm{~Hz}$ ). The CompCor method (Behzadi et al., 2007) as implemented in the DPABI toolbox (http://rfmri.org/dpabi) was used to estimate sources of noise within the white matter and cerebrospinal fluid masks on the resting-state data. The first five principal components together with six head motion parameters obtained from preprocessing were used for nuisance regression (cf. Chai et al., 2012; Long et al., 2016). The two resting-state sequences were preprocessed separately and then both included in the second level group analyses.

\subsubsection{Network Analysis}

Eigenvector centrality mapping (ECM) was used as an established graph-theoretical network approach that aims to identify "hubs" that are central or prominent within a network, similar to the "PageRank" algorithm of Google (Lohmann et al., 2010; Wink et al., 2012). ECM analyses were carried out using fastECM software (Wink et al., 2012). Individual threedimensional maps for each participant and each condition were obtained including voxel-

wise measures of eigenvector centrality (Lohmann et al., 2010; Zuo et al., 2012). Briefly, ECM quantifies the correlation of each voxel in the brain with other voxels, taking into account how they are themselves connected across the brain (Lohmann et al., 2010). Thus, ECM values reflect the impact or relevance (i.e., centrality) of a region in the entire brain network. Importantly, ECM analysis does not require any a priori assumptions (Bonacich, 
2007) - avoiding hypotheses about anatomical network organization or the involvement of particular brain regions - and therefore allows for a data-driven explorative approach (Zuo et al., 2012).

ECM analyses were restricted to a binarized grey matter mask. The cerebellum was excluded from the mask due to insufficient scanning range of the MRI resulting in an incomplete cerebellum in the field of view in the majority of participants (see also Luo et al., 2016).

\subsubsection{Seed-based Analysis}

Seed-based analyses were conducted in order to further investigate the origin of tDCSinduced centrality changes (Marangolo et al., 2016; Nierhaus et al., 2015; Taubert et al., 2011) and to explore the changes in functional connectivity of the stimulation site. Therefore, we used the most significant cluster from ECM analysis (peak coordinates: $x=-6, y=-44, z$ $=59 ; k=325, T=5.22$ ) which corresponded to the brain area underneath the "active" electrode as the seed region. Given that a significant interaction of anodal stimulation condition and age group was found (see below), we aimed to further explore the differences in functional connectivity patterns of this region. Therefore, the temporal correlation of the time-series between this seed region and all other voxels in the brain was computed in each individual scan, separately for anodal and sham stimulation conditions.

\subsubsection{Voxel-based morphometry}

Voxel-based morphometry was performed using the CAT12 toolbox for SPM12 (http://dbm.neuro.uni-jena.de/cat/) in order to compare grey matter volume between age groups. We used default settings described in the manual (http://dbm.neuro.unijena.de/cat12/CAT12-Manual.pdf), including normalization to MNI template, structural segmentation, bias correction, and smoothing with a Gaussian filter (8 $\mathrm{mm}$ full-width halfmaximum).

\subsubsection{MRI Statistics}


To investigate the interaction between stimulation condition and age group, repeatedmeasures ANOVAs were conducted using a flexible factorial design as implemented in SPM12 (Gläscher and Gitelman, 2008). For the analysis of EC changes, the main contrast of interest was [(anodal old $>$ sham old $) \neq$ (anodal young $>$ sham young)], or [(cathodal old $>$ sham old) $\neq$ (cathodal young $>$ sham young)], respectively, which reflects the difference of stimulation effects between the two age groups. For illustration purposes, we extracted ECM values from significant clusters from the interaction contrast using the MarsBaR toolbox (http://marsbar.sourceforge.net). Seed-based functional connectivity was compared between anodal and sham stimulation condition in both age groups with a flexible factorial model testing for the main effect of tDCS. Grey matter volume differences were tested with a twosample t-test comparing whole-brain grey matter volume between age groups, adjusted for total intracranial volume. Neuroelf Version 1.1 was used for data thresholding and visualization (http://neuroelf.net). Cluster-extent thresholds were determined with Monte Carlo simulation using the AlphaSim procedure (Forman et al., 1995) using a family-wise error (FWE) cluster level correction of $p_{\mathrm{FWE}}<0.05$. All second-level analyses were restricted to a binarized grey matter mask.

\subsection{Non-imaging statistics}

All other statistical analyses were performed using IBM SPSS Statistics 24 (http://www01.ibm.com/software/uk/analytics/spss/). Linear mixed models were computed for the comparison of positive and negative affect changes in stimulation conditions. Models included the factor stimulation condition (anodal, cathodal, sham), group (old, young) and time (pre, post), where appropriate as well as their interactions. Logistic mixed models were computed for each adverse effect in order to compare the amount of participants who reported its occurrence in the stimulation conditions. Models included the factors stimulation condition and age group and were adjusted for gender. A two-sided significance level of $\alpha=$ 0.05 was used.

\section{Results}




\subsection{Electric field simulations}

The modeling results indicate that this conventional electrode montage induced electric fields with maximum intensity between the two electrodes, but also high intensities around the intended target area. More specifically, in both age groups, high electric field intensities occurred in the vicinity of the left-hemispheric paracentral lobe, at the precentral gyrus and central sulcus (Fig. 1A). On average, the spatial distribution of the electric field strength is highly similar between age groups, with slightly more frontal stimulation at bilateral superior and middle frontal sites (including the precentral gyrus) in young adults. However, the standard deviation of the field strength suggests a higher inter-subject variability at these frontal and paracentral sites in older adults, indicating that the field intensities and/or spatial distribution may be more inconsistent within the older group compared to the young group (Fig. 1B). Visualizing the component of the electric field vector that is normal (i.e., orthogonal) to the cortical sheet (Fig. 1C) indicates that anodal stimulation is most likely induced at left-hemispheric paracentral lobes, including precentral and postcentral gyri and maximum intensity at the central sulcus. The spatial distributions of the group-averaged normal components were similar for both age groups. 
A old

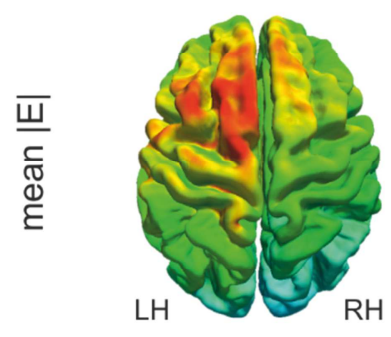

B

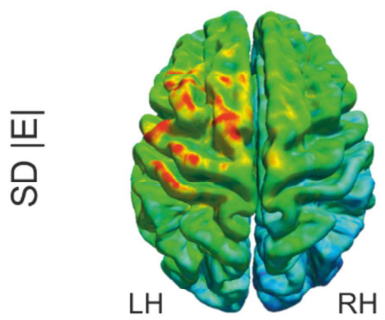

C

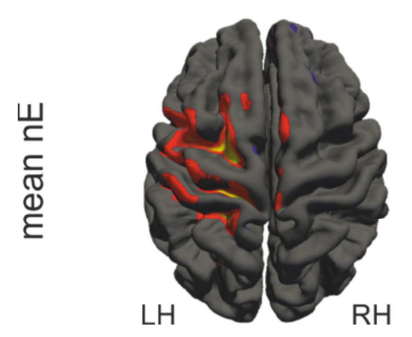

old
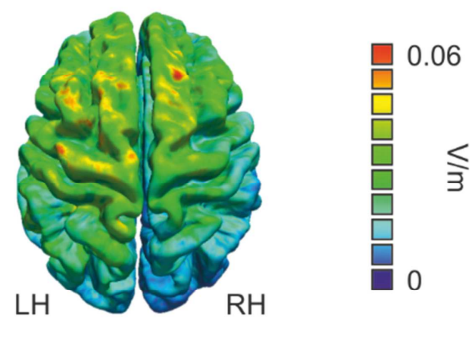

young
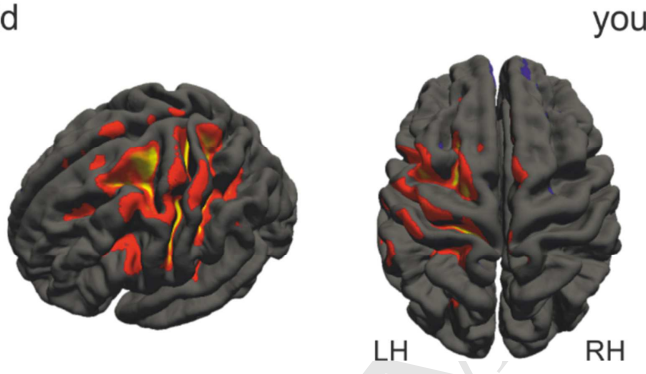

young
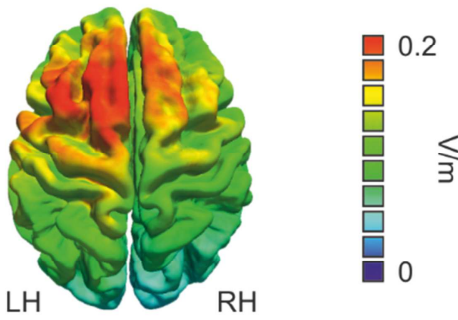

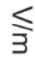
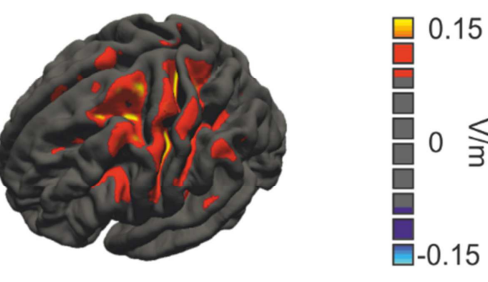

Figure 1. Electric field distribution for the anodal stimulation condition derived from FEM calculations using SimNIBS. A: Electric field strength $(|E|)$ averaged across older (left panel) and young adults (right panel). B: Variability of the electric field strength (standard deviation) among older (left panel) and young adults (right panel). C: Component of the electric field that is directed orthogonally to the cortical sheet (termed "normal" component, $\mathrm{nE}$ ), averaged across older (left panel) and young adults (right panel). Positive values (red-yellow) indicate anodal stimulation, negative values (violet-blue) indicate cathodal stimulation. $\mathrm{LH}$, left hemisphere. $\mathrm{RH}$, right hemisphere.

\subsection{Age group-dependent differences in network centrality}

Mean network centrality maps acquired in the sham stimulation condition are illustrated in Figure 2. Older compared to younger adults showed both reduced and increased ECM values in various brain regions (Fig. 3, Tab. 1). Specifically, ECM values in older adults were reduced in bilateral occipital (including lingual and fusiform gyri), bilateral medial frontal (cingulate gyrus), left hemisphere (dorsomedial) postcentral and right hemisphere temporoparietal gyri (including posterior insula/parietal operculum and middle temporal/supramarginal gyrus). Significant centrality increases emerged in left lateral and medial temporoparietal areas (including superior parietal lobule, precuneus and 
caudate/parahippocampal gyrus), left hemisphere (ventrolateral) postcentral gyrus and bilateral anterior insula.

A old

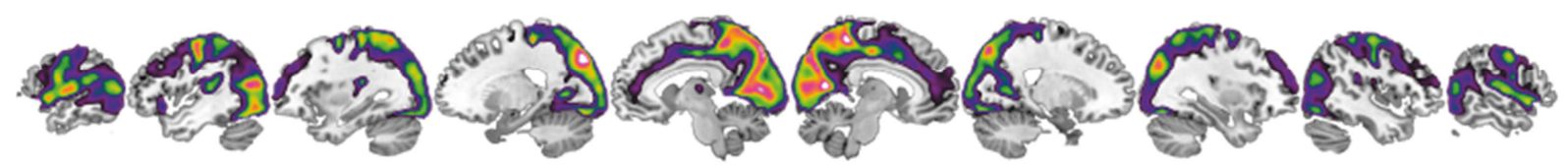

B young

$\mathrm{LH}$

$\mathrm{RH}$

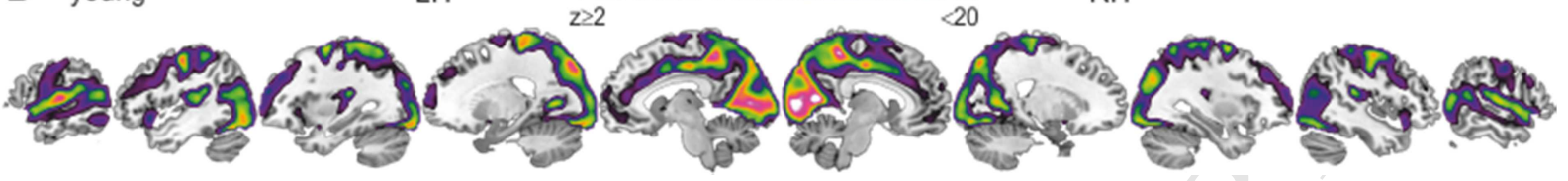

Figure 2. Mean ECM maps in sham stimulation condition in older (A) and young adults (B). Whole-brain voxel-wise mean EC values were generated from all individual maps in each group and transformed into $z$-values. The scale displays values with intermediate (violet) to high centrality values (white). LH, left hemisphere. $\mathrm{RH}$, right hemisphere.

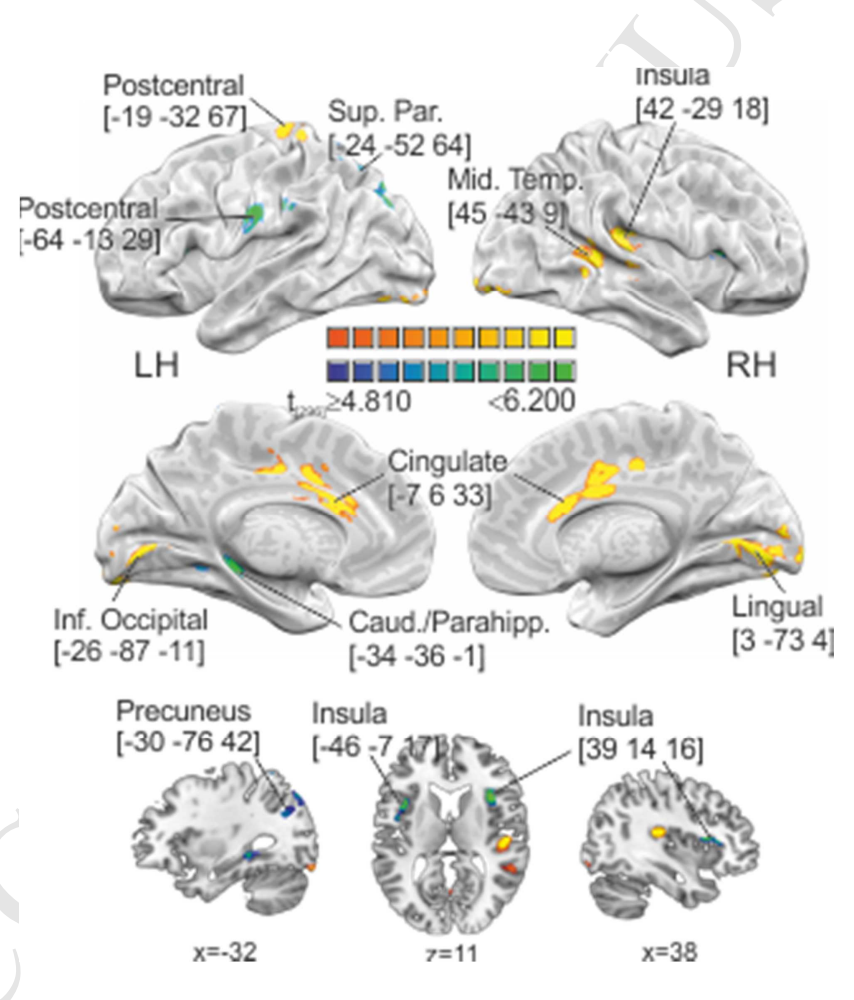

Figure 3. Comparison of ECM values between age groups in sham stimulation condition. Whole brain analyses for the contrast [(old) $\neq$ (young)]. This "group" main effect shows elevated centrality in young compared to older adults (in red-yellow) and elevated centrality in older compared to young adults (in blue-green). Clusters were significant at $p_{\mathrm{FWE}}<0.05$ ( $k=1,080 \mathrm{~mm}^{3} / 40$ voxel). LH, left hemisphere. $\mathrm{RH}$, right hemisphere.

Table 1. Comparison of ECM values between older and young adults in sham.

\begin{tabular}{llllll}
\hline Brain region & Hemi & BA & $\begin{array}{l}\text { Coordinates } \\
x\end{array}$ & $k$ & $T$ \\
\hline
\end{tabular}

Older adults $<$ young adults 


\section{ACCEPTED MANUSCRIPT}

Lingual Gyrus

Inferior Occipital / Fusiform Gyrus

Insula / Parietal Operculum Cortex

Postcentral Gyrus

Cingulate Gyrus

Lingual Gyrus / Occipital Pole

Middle Temporal / Supramarginal Gyrus

Older adults $>$ young adults

Insula / Central Opercular Cortex

Superior Parietal Lobule

Insula / Frontal Operculum Cortex

Precuneus

Caudate / Parahippocampal Gyrus

Postcentral Gyrus

\begin{tabular}{llrrrrr}
$\mathrm{RH}$ & $17 / 18,30$ & 3 & -73 & 4 & 114 & 9.78 \\
$\mathrm{LH}$ & 18 & -26 & -87 & -11 & 83 & 8.25 \\
$\mathrm{RH}$ & $13,22,41$ & 42 & -29 & 18 & 116 & 8.08 \\
$\mathrm{LH}$ & 3 & -19 & -32 & 67 & 44 & 7.45 \\
$\mathrm{LH} / \mathrm{RH}$ & 24,32 & -7 & 6 & 33 & 142 & 7.12 \\
$\mathrm{RH}$ & 18 & 24 & -94 & -5 & 70 & 6.72 \\
$\mathrm{RH}$ & $21 / 22$ & 45 & -43 & 9 & 47 & 6.37 \\
& & & & & & \\
$\mathrm{LH}$ & 13,45 & -46 & -7 & 17 & 40 & -8.10 \\
$\mathrm{LH}$ & 7,40 & -24 & -52 & 64 & 43 & -7.17 \\
$\mathrm{RH}$ & 13,45 & 39 & 14 & 16 & 41 & -7.17 \\
$\mathrm{LH}$ & 19,39 & -30 & -76 & 42 & 72 & -6.53 \\
$\mathrm{LH}$ & 19,30 & -34 & -36 & -1 & 40 & -6.28 \\
$\mathrm{LH}$ & 3,40 & -64 & -13 & 29 & 41 & -5.99 \\
\hline
\end{tabular}

Anatomical location of cluster peaks, FWE-corrected ( $p_{\text {FWE }}<0.05, k>1,080 \mathrm{~mm}^{3 / 40}$ voxel). Coordinates of peak voxel $(x, y, z$ in $\mathrm{mm}$ ) in Talairach space. Hemi, hemisphere ( $\mathrm{LH}$, left hemisphere; $\mathrm{RH}$, right hemisphere); $\mathrm{BA}$, Brodmann areas; $k$, cluster extent (number of voxels); $\mathrm{T}$, $\mathrm{t}$-value of peak.

\subsection{Grey matter volume differences}

In order to explore the overlap of group differences in EC values with grey matter volume differences, we applied an approach similar to that described by Binnewijzend et al. (2014).

Figure 4 depicts the spatial distribution of grey matter atrophy in older compared to young adults. Differences were visible across the whole-brain grey matter, prominently in the ventromedial prefrontal and bilateral temporoparietal cortices. The clusters where EC values were decreased and increased in older compared to young adults partly overlapped with those regions that showed lower grey matter volume (271 voxel / $7317 \mathrm{~mm}^{3}$, and 69 voxel / $1863 \mathrm{~mm}^{2}$, respectively). This corresponds to a total overlap of connectivity differences and grey matter differences between age groups of $38 \%$ (340 out of 893 voxel).

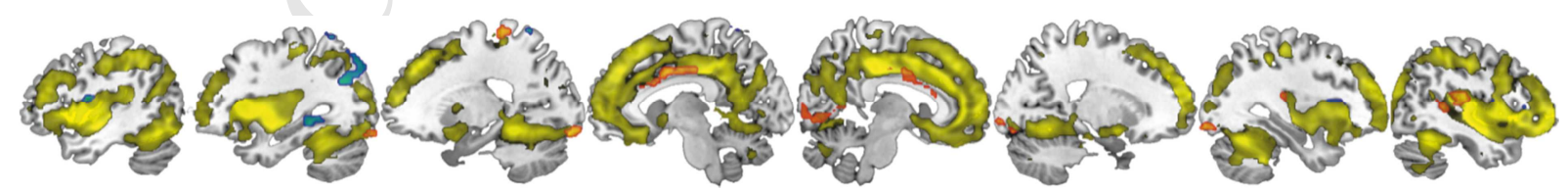

Figure 4. Age-group grey matter volume differences (yellow: regions of decreased grey matter volume in older compared to young adults; $p_{\mathrm{FWE}}<0.05, k=40$ ) overlaid with age-group EC differences (red: clusters of decreased EC values, blue: increased EC values in older adults; $p_{\mathrm{FWE}}<0.05, k=40$ ).

\subsection{Age-group dependent effects of anodal tDCS}




\section{ACCEPTED MANUSCRIPT}

ECM analyses revealed a significant interaction between anodal stimulation condition and age group as illustrated in Figure 5 and Table 2. Five significant clusters emerged for the contrast $[$ (anodal old $>$ sham old $) \neq$ (anodal young $>$ sham young $)]$ demonstrating that anodal stimulation resulted in different effects on eigenvector centrality in left- and right-hemispheric paracentral lobuli, left and right precuneus, right superior parietal lobule and right inferior parietal gyrus in older versus young adults. Conditional main effects within each group showed reduced EC values during anodal compared to sham stimulation in the left postcentral gyrus in older adults. In young adults, EC values in a cluster including the left precuneus and postcentral gyrus were increased during anodal compared to sham stimulation, while EC values in the right inferior parietal gyrus were decreased (Fig. S1, Table 2). No significant effects were found for cathodal stimulation. 
A Interaction condition $\times$ group

[(anodal old > sham old $) \neq$ (anodal young $>$ sham young $)]$
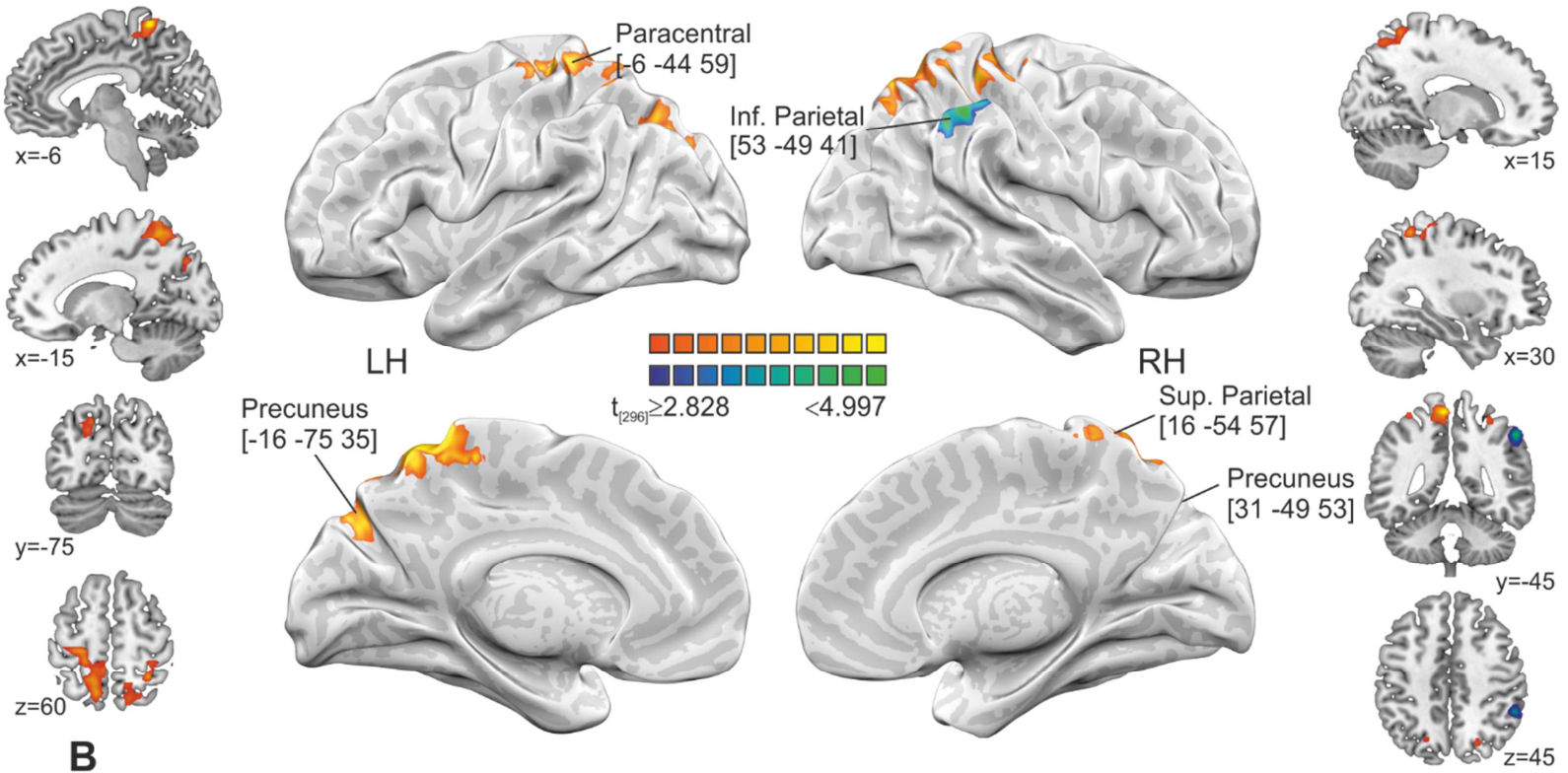

B

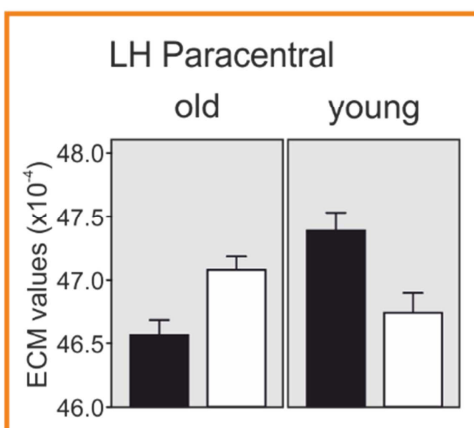

RH Precuneus
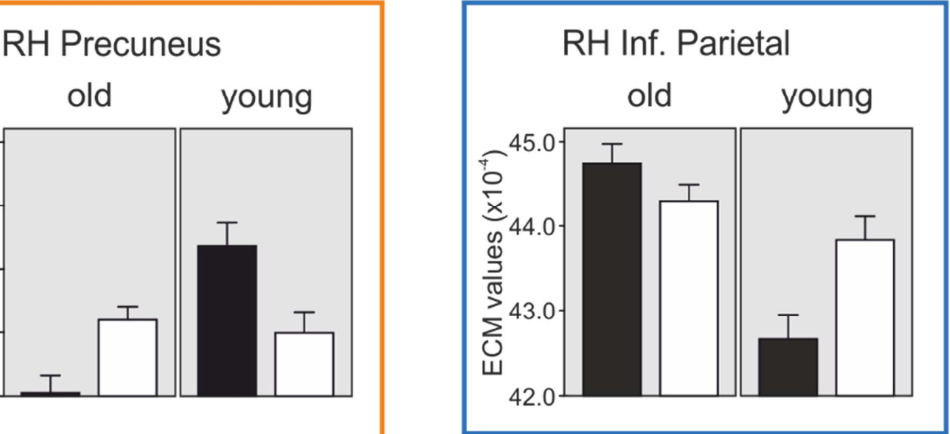

RH Sup. Parietal

LH Precuneus
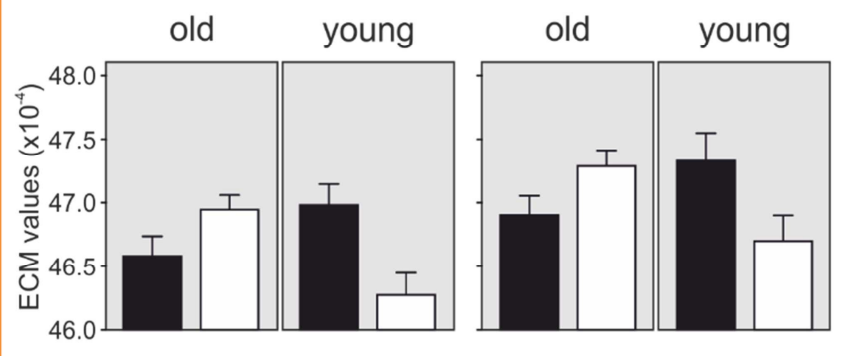

anodal

sham

Figure 5. Effect of anodal compared to sham stimulation condition in older and young adults on ECM values. A: Whole brain analyses for the contrast [(anodal old > sham old) $\neq$ (anodal young > sham young)]. This "condition x group" interaction shows elevated centrality under anodal (minus sham) stimulation condition in young compared to older adults in red-yellow and elevated centrality under anodal (minus sham) stimulation condition in older compared to young adults in blue-green. Cluster-extent thresholds were calculated using Monte Carlo simulation with AlphaSim, resulting in $k=$ 63 for $p_{\mathrm{u}}<0.005$ which corresponds to $p_{\mathrm{FWE}}<0.05$ (family-wise error correction for multiple comparisons). B: ECM values in significant clusters extracted from the "condition $\mathrm{x}$ group" interaction. Means and standard errors of ECM values $\left(\times 10^{-4}\right)$ in the left paracentral lobule, right precuneus, left superior parietal lobule, left precuneus, and right inferior parietal lobule clusters are shown for each group and condition (black bars: anodal, white bars: sham). Arrows illustrate the direction of "change" relative to sham stimulation in each age group (orange arrows: decrease in old / increase in young, blue arrows: increase in old / decrease in young). $\mathrm{LH}$, left hemisphere. $\mathrm{RH}$, right hemisphere. 
Table 2. Comparison of anodal stimulation effects on ECM values.

\begin{tabular}{|c|c|c|c|c|c|c|c|}
\hline \multirow[t]{2}{*}{ Brain region } & \multirow[t]{2}{*}{ Hemi } & \multirow[t]{2}{*}{ BA } & \multicolumn{3}{|c|}{ Coordinates } & \multirow[t]{2}{*}{$k$} & \multirow[t]{2}{*}{$\mathrm{T}$} \\
\hline & & & $\mathrm{x}$ & $\mathrm{y}$ & $z$ & & \\
\hline \multicolumn{8}{|c|}{ [(anodal old > sham old $) \neq$ (anodal young $>$ sham young $)]$} \\
\hline Paracentral Lobule & $\mathrm{LH}$ & $3-5$ & -6 & -44 & 59 & 325 & 5.22 \\
\hline Precuneus & $\mathrm{RH}$ & 7 & 31 & -49 & 53 & 82 & 4.42 \\
\hline Superior Parietal Lobule & $\mathrm{RH}$ & 7 & 16 & -54 & 57 & 103 & 4.05 \\
\hline Precuneus & $\mathrm{LH}$ & 7,19 & -16 & -75 & 35 & 110 & 3.87 \\
\hline Inferior Parietal Lobule & $\mathrm{RH}$ & 40 & 53 & -49 & 41 & 69 & -4.41 \\
\hline \multicolumn{8}{|l|}{ Older adults (anodal $\neq$ sham) } \\
\hline Postcentral Gyrus & $\mathrm{LH}$ & 3,5 & -35 & -44 & 56 & 103 & -4.36 \\
\hline \multicolumn{8}{|l|}{ Young adults (anodal $\neq$ sham) } \\
\hline Precuneus / Postcentral Gyrus & $\mathrm{LH}$ & $3,5,7$ & -9 & -47 & 62 & 105 & 4.36 \\
\hline Inferior Parietal Lobule & $\mathrm{RH}$ & 40 & 50 & -49 & 42 & 80 & -4.02 \\
\hline
\end{tabular}

Anatomical location of cluster peaks, FWE-corrected (condition $\times$ group interaction: $p_{\mathrm{FWE}}\langle 0.05, k\rangle$ $1,701 \mathrm{~mm}^{3} / 63$ voxel; main effect older adults: $p_{\mathrm{FWE}}<0.05, k>1,674 \mathrm{~mm}^{3} / 62$ voxel; main effect young adults: $\left.k>1,755 \mathrm{~mm}^{3} / 65\right)$. Coordinates of peak voxel $(x, y, z$ in $\mathrm{mm})$ in Talairach space. Hemi, hemisphere ( $\mathrm{LH}$, left hemisphere; $\mathrm{RH}$, right hemisphere); $\mathrm{BA}$, Brodmann areas; $k$, cluster extent (number of voxels); $\mathrm{T}$, $\mathrm{t}$-value of peak.

Whole-brain seed-based functional connectivity analyses were calculated with the seed in the left paracentral lobule and are shown separately for anodal and sham stimulation condition in both age groups in Figure 6. The maps are mainly composed by the sensorimotor network including brain areas surrounding the seed region, homologue areas in the right hemisphere and medial brain areas including precentral, postcentral gyri and juxtapositional lobule cortex.

A old
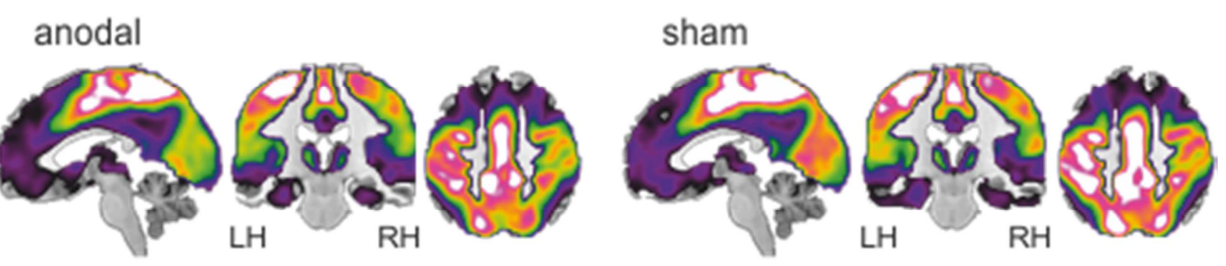

B young anodal
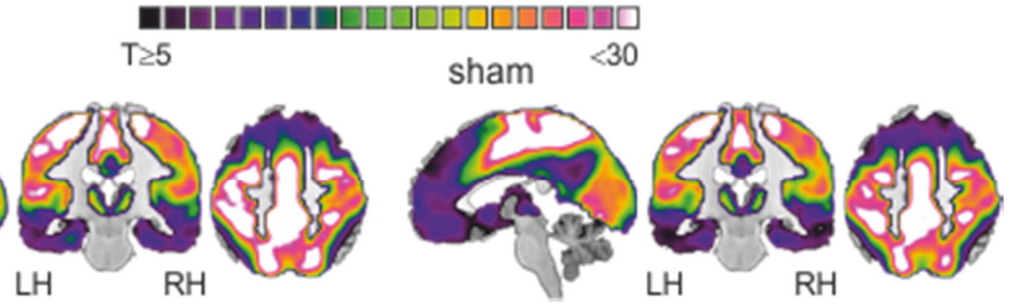

Figure 6. Mean functional connectivity maps with seed in LH paracentral gyrus in anodal and sham stimulation conditions in older $(A)$ and young adults $(B)$. $M N I$ coordinates of slice positions are $(x=-4, y=-22, z=51)$. $L H$, left hemisphere. $R H$, right hemisphere. 
Seed-based analyses revealed significant functional connectivity decreases in older but increases in young adults, during anodal compared to sham stimulation (Fig. 7, Table 3). Older adults exhibited functional connectivity decreases between left paracentral lobule and bilateral lingual gyri (including cuneus, posterior cingulate, precuneus, and fusiform gyri), left middle occipital (including cuneus, but also precuneus and middle temporal gyrus), left postcentral gyrus (including inferior parietal lobule) and right posterior cingulate (including parahippocampal gyrus). Young adults showed increases in functional connectivity between the left paracentral lobule and bilateral anterior cingulate (including superior and medial frontal gyrus), left cingulate and right precuneus.
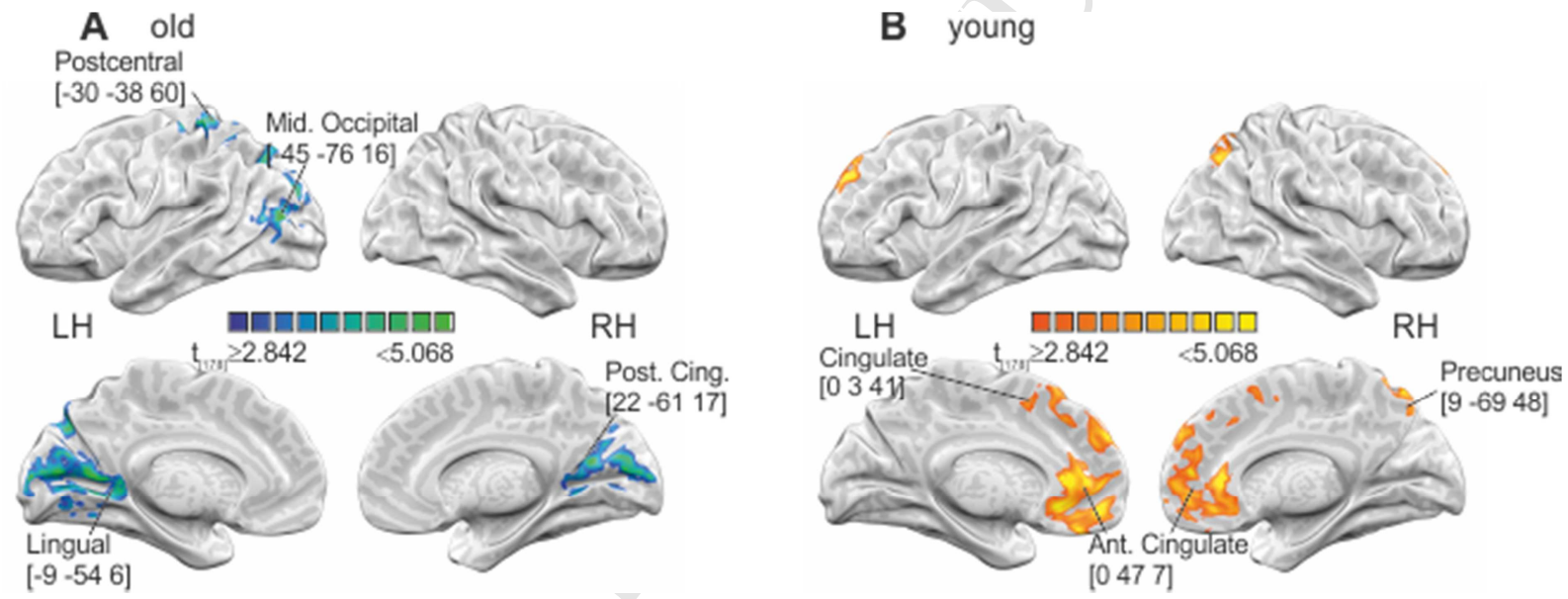

Figure 7. Seed-based functional connectivity during anodal compared to sham stimulation with seed in LH paracentral gyrus [(anodal $\neq$ sham)]. A: In older adults. Clusters in blue-green depict decreased correlations to the seed during anodal stimulation. Cluster-extent thresholds were calculated using Monte Carlo simulation with AlphaSim, resulting in $k=77$ for $p_{u}<0.005$ which corresponds to $p_{\mathrm{FWE}}<0.05$ (family-wise error correction for multiple comparisons). B: In young adults. Clusters in red-yellow depict increased correlations to the seed during anodal stimulation. Clusterextent thresholds were calculated using Monte Carlo simulation with AlphaSim, resulting in $k=84$ for $p_{\mathrm{u}}<0.005$ which corresponds to $p_{\mathrm{FWE}}<0.05$ (family-wise error correction for multiple comparisons). $\mathrm{LH}$, left hemisphere. $\mathrm{RH}$, right hemisphere.

Table 3. Clusters showing a significant connectivity modulation with the left paracentral lobule.

\begin{tabular}{|c|c|c|c|c|c|c|c|}
\hline \multirow[t]{2}{*}{ Brain region } & \multirow[t]{2}{*}{ Hemi } & \multirow[t]{2}{*}{ BA } & \multicolumn{3}{|c|}{ Coordinates } & \multirow[t]{2}{*}{$k$} & \multirow[t]{2}{*}{$\mathrm{T}$} \\
\hline & & & $\mathrm{x}$ & $\mathrm{y}$ & z & & \\
\hline \multicolumn{8}{|c|}{ [(anodal old < sham old $) \neq$ (anodal young $>$ sham young $)]$} \\
\hline Superior Frontal Gyrus & $\mathrm{LH} / \mathrm{RH}$ & $8 / 9,10 / 11,24,32$ & -22 & 52 & 32 & 788 & 5.12 \\
\hline Precuneus & $\mathrm{LH} / \mathrm{RH}$ & $4 / 5,7,29-31,18 / 19,39 / 40$ & -19 & -67 & 38 & 2112 & 5.10 \\
\hline Inferior Frontal Gyrus & $\mathrm{LH}$ & 47 & -42 & 21 & -11 & 84 & 4.20 \\
\hline Postcentral Gyrus & $\mathrm{LH}$ & $3 / 4,6,9$ & -47 & -14 & 52 & 253 & 3.99 \\
\hline
\end{tabular}




\begin{tabular}{|c|c|c|c|c|c|c|c|}
\hline Middle Frontal Gyrus & $\mathrm{RH}$ & $3 / 4,6$ & 39 & -1 & 50 & 82 & 3.83 \\
\hline \multicolumn{8}{|l|}{ Older adults (anodal $\neq$ sham) } \\
\hline Lingual Gyrus & $\mathrm{LH} / \mathrm{RH}$ & $17-19,30$ & -9 & -54 & 6 & 416 & -4.46 \\
\hline Postcentral Gyrus & $\mathrm{LH}$ & $3,5,7,40$ & -30 & -38 & 60 & 101 & -4.40 \\
\hline Middle Occipital Gyrus & $\mathrm{LH}$ & 7,19 & -45 & -76 & 16 & 284 & -4.32 \\
\hline Posterior Cingulate Gyrus & $\mathrm{RH}$ & $18,30 / 31$ & 22 & -61 & 17 & 110 & -4.19 \\
\hline \multicolumn{8}{|l|}{ Young adults (anodal $\neq$ sham) } \\
\hline Anterior Cingulate & $\mathrm{LH} / \mathrm{RH}$ & $8-11,24,32$ & 0 & 47 & 7 & 639 & 4.66 \\
\hline Precuneus & $\mathrm{RH}$ & 7 & 9 & -69 & 48 & 90 & 3.99 \\
\hline Cingulate Gyrus & $\mathrm{LH}$ & $6,8,24,32$ & 0 & 3 & 41 & 96 & 3.62 \\
\hline
\end{tabular}

Anatomical location of cluster peaks, FWE-corrected (condition $x$ group interaction: $p_{\mathrm{FWE}}<0.05, k>$ $2,106 \mathrm{~mm}^{3} / 78$ voxel; main effect older adults: $p_{\mathrm{FWE}}<0.05, k>2,079 \mathrm{~mm}^{3} / 77$ voxel; main effect young adults: $\left.k>2,268 \mathrm{~mm}^{3} / 84\right)$. Coordinates of peak voxel ( $x, y, z$ in $\mathrm{mm}$ ) in Talairach space. Hemi, hemisphere ( $\mathrm{LH}$, left hemisphere; $\mathrm{RH}$, right hemisphere); $\mathrm{BA}$, Brodmann areas; $k$, cluster extent (number of voxels); T, t-value of peak.

\subsection{Mood ratings and adverse effects}

Positive and negative affect before and after scanning are displayed in Table 4. There were no significant differences between stimulation conditions $\left(F_{(2,296)}=0.66, p=0.517\right.$ for positive and $F_{(2,296)}=0.83, p=0.437$ for negative affect). A significant group effect $\left(F_{(1,58)}=5.55, p=\right.$ 0.022 for positive and $F_{(1,58)}=5.04, p=0.029$ for negative affect) indicated that older adults reported more positive (mean difference [95\%-CI]: 0.419 [0.063, 0.775]) and less negative mood (mean difference [95\%-Cl]: -1.121 [-0.229, -0.013]).

Most common adverse effects were tingling and fatigue which were reported by $52 \%$, and $40 \%$, respectively, of all participants. Number of participants who reported occurrence of the respective adverse effects are presented in Table 5. No significant stimulation effect emerged for adverse effects (all $p$-values $>0.05$ ). Significant main effects of age group were found for pain $(p=0.040)$, burning sensation $(p=0.037)$ and loss of concentration $(p<$ 0.001). Therefore, older participants reported overall slightly more pronounced adverse effects than young participants, but this was independent of stimulation condition.

Table 4. Mood ratings before and after MRI session.

\begin{tabular}{lcccccc}
\hline & \multicolumn{2}{c}{ Anodal } & & \multicolumn{2}{c}{ Cathodal } & \multicolumn{2}{c}{ Sham } \\
& old & young & old & young & old & young \\
\hline Positive affect & & & & & &
\end{tabular}




\begin{tabular}{lllllll} 
Pre & $3.30(0.78)$ & $2.91(0.57)$ & $3.27(0.75)$ & $2.77(0.61)$ & $3.24(0.75)$ & $2.72(0.60)$ \\
Post & $3.06(1.04)$ & $2.62(0.59)$ & $3.10(0.94)$ & $2.62(0.64)$ & $2.94(0.96)$ & $2.76(0.74)$ \\
Negative affect & & & & & & \\
$\quad$ Pre & $1.14(0.23)$ & $1.28(0.40)$ & $1.21(0.33)$ & $1.26(0.30)$ & $1.16(0.18)$ & $1.32(0.39)$ \\
Post & $1.08(0.14)$ & $1.17(0.21)$ & $1.07(0.13)$ & $1.21(0.33)$ & $1.09(0.18)$ & $1.24(0.35)$ \\
\hline
\end{tabular}

Mean (SD) values.

Table 5. Adverse effects during stimulation.

\begin{tabular}{lcccccc}
\hline & \multicolumn{3}{c}{ Anodal } & \multicolumn{2}{c}{ Cathodal } & \multicolumn{2}{c}{ Sham } \\
& old & young & old & young & old & young \\
\hline Pain & 2 & 12 & 5 & 8 & 3 & 7 \\
Tingling & 18 & 12 & 19 & 12 & 18 & 9 \\
Itchiness & 4 & 4 & 4 & 5 & 2 & 3 \\
Burning & 3 & 12 & 3 & 7 & 3 & 6 \\
Fatigue & 7 & 14 & 8 & 13 & 8 & 15 \\
Tension & 2 & 8 & 5 & 4 & 3 & 5 \\
Loss of concentration & 0 & 4 & 0 & 4 & 0 & 5 \\
Headache & 0 & 1 & 1 & 0 & 1 & 1 \\
Discomfort & 5 & 10 & 7 & 8 & 5 & 7 \\
\hline
\end{tabular}

Number of participants within respective age group (total $n=30$ in each group).

\section{Discussion}

The present study explored the difference in tDCS effects on functional connectivity between older and young adults. We used a counterbalanced, cross-over design with anodal, cathodal and sham stimulation targeting the sensorimotor network. Functional network analysis revealed neuromodulatory effects of tDCS in brain regions under the anode, but also distant to the stimulation site. We observed differential effects of anodal stimulation on centrality in left paracentral, right superior parietal gyri, bilateral precuneus and right inferior parietal lobule in older versus young adults. Older adults showed decreased eigenvector centrality in the left postcentral gyrus in anodal compared to sham stimulation. Young adults showed centrality increases in left precuneus and postcentral gyrus while centrality in the right inferior parietal gyrus was decreased in anodal compared to sham stimulation. Complementary seed-based functional connectivity analyses assessing voxel-wise correlation of the stimulated left sensorimotor cortex with the rest of the brain showed that the neuromodulatory tDCS effects may originate from an increased coupling with 
ventromedial prefrontal brain areas in young adults, and from an increased decoupling with posteromedial brain areas in older adults.

This is the first study that included both older and young adults to systematically examine the effects of non-invasive brain stimulation on task-independent resting-state functional network connectivity. ECM analysis investigated the global organization of whole brain network architecture by quantifying the relevance of specific nodes within the global network without a priori specification of involved regions or networks (Bullmore and Sporns, 2009; Lohmann et al., 2010). Increased eigenvector centrality values reflect stronger connections of the respective area, i.e., augmented synchronization, with other brain areas that are themselves highly connected (Lohmann et al., 2010; Wink et al., 2012). Importantly, as all connections within the grey matter are taken into account, interpretation of local changes should be relative and not independent of the global pattern as increased eigenvector centrality values in specific areas may go along with decreases in other areas (van Duinkerken et al., 2017; Wink et al., 2012). ECM has been shown to be sensitive to both short- and long-range connections and to subtle network architecture alterations and is therefore well suited for the exploratory comparison of modulations through experimental conditions (Binnewijzend et al., 2014; van Duinkerken et al., 2017; Wink et al., 2012).

Previous studies have used ECM to describe network architecture changes between patient groups and healthy individuals (Adriaanse et al., 2016; in Alzheimer's disease: Binnewijzend et al., 2014; in Multiple Sclerosis: Eijlers et al., 2017; in Type 1 Diabetes Mellitus: van Duinkerken et al., 2017) or between tDCS conditions (Lindenberg et al., 2013; Lindenberg et al., 2016; Meinzer et al., 2012; Meinzer et al., 2015; Sehm et al., 2012). High similarity between mean centrality maps of different groups and conditions - which we confirm with our data - reflects a strong reliability of the patterns (Wink et al., 2012). We observed the highest eigenvector centrality values in occipital and precuneus/posterior cingulate cortex. This is consistent with the literature, denoting these posterior medial regions as most important hubs in the global brain network (Binnewijzend et al., 2014; Eijlers et al., 2017; Zuo et al., 2012). In 
our group of older adults, highest eigenvector centrality values were spread across the medial frontal cortex compared to young adults, similar to what has been described for patients with Alzheimer's disease compared to healthy controls (Binnewijzend et al., 2014). The comparison of centrality values between older and young adults revealed an age-related reorganization of the relative importance of particular hubs in the global network. In line with our previous study (Lindenberg et al., 2013), older adults exhibited lower occipital, medial frontal, left sensorimotor and right posterior temporoparietal connectivity and higher left lateral and medial temporoparietal, left ventrolateral postcentral and bilateral insula connectivity. Age-related functional network reorganization has been widely described, including both increases and decreases and associations with cognitive scores (Geerligs et al., 2015; Sala-Llonch et al., 2015). The observed age-dependent neural patterns in our study corroborate previous descriptions of an adaptive posterior-anterior shift in brain activity and brain functional network dynamics in healthy aging (Davis et al., 2008; Dennis and Cabeza, 2008; Zhang et al., 2017). These age-related connectivity alterations which include default mode network regions were observed to be accelerated in Alzheimer's disease (AD) (Jones et al., 2011; Wiepert et al., 2017). Coherently, highly connected posterior occipital and parietal network hubs were found to be related to age-related cognitive decline and to be most vulnerable in AD (Adriaanse et al., 2016; Binnewijzend et al., 2014). In sum, our data confirms previously observed centrality patterns in the whole brain network and their modulation by age.

Of note, age-related grey matter atrophy may be at least partly responsible for BOLD fMRI activity and connectivity differences and affect the distribution of current density (Johnson et al., 2000; Opitz et al., 2015). In our data, approximately one third of clusters where we found significant age group connectivity differences overlapped with brain areas where grey matter volume was significantly lower in older adults. This is in line with previous evidence reporting a relationship of cerebral atrophy with functional activity and connectivity (Agosta et al., 2012; Binnewijzend et al., 2014; Johnson et al., 2000). Further, computational modelling approaches have suggested that individual anatomy is associated with differences in current 
field distribution during tDCS (Kim et al., 2014; Laakso et al., 2015). Our modeling results showed highly similar spatial distributions and intensities of the induced electric fields as well as consistent field directions in both age groups. In line with previous computational modeling studies, strong fields occurred underneath the anode with maximum intensity between the two electrodes (Datta et al., 2009; Laakso et al., 2015; Opitz et al., 2015; Polania et al., 2018; Saturnino et al., 2015). The higher variability of the electric field in the older group indicated that stimulation intensities may be more inconsistent within the group of older compared to young adults. The variability in induced electric fields may be linked to inconsistent neurophysiological effects of tDCS (Laakso et al., 2015). Increased age and decreased grey matter volume (resulting in more CSF) may lead to less current entering the brain (Laakso et al., 2015; Mahdavi et al., 2018; Opitz et al., 2015). However, Thomas et al. described that both reduced global field peaks due to "shunting" away current by increased CSF but also increased field values due to "wide pockets" of CSF that attract current to specific brain regions may be conceivable consequences of brain atrophy (Thomas et al., 2017). Together, these modeling studies suggest that age-related structural deterioration may affect patterns of current flow in older adults, but also highlight that these changes may be quite complex and non-linear (Laakso et al., 2015; Thomas et al., 2017). Importantly, although computational modeling approaches clearly advance our understanding of NIBSinduced neural effects, validation studies are still required determining the predictive validity of modelling results for individual neural and behavioral outcomes (Hartwigsen et al., 2015; Mahdavi et al., 2018; Polania et al., 2018). In order to disentangle effects of age and associated grey matter atrophy, longitudinal and multimodal data are required.

Regarding stimulation effects, our data support and extend previous observations of widespread rather than local stimulation-induced functional connectivity alterations through sensorimotor tDCS (Polanía et al., 2011; Sehm et al., 2012). Previous studies investigating either young or older adults have suggested differential stimulation effects on resting-state fMRI (Antonenko et al., 2017; Bachtiar et al., 2015; Lindenberg et al., 2013; Lindenberg et al., 2016), but no study to date has systematically compared these effects. Using the same 
electrode configuration, but stimulating during task fMRI, the first study that included both age groups to examine neural network effects of tDCS-induced performance improvement was conducted by Martin et al. (2017). Anodal tDCS not only resulted in improved task performance, but also in similar network-level modulations across both age groups, as evident from independent component analysis. In older adults only, anodal tDCS additionally increased frontoparietal network laterality. The authors concluded that age-associated baseline differences in network architecture may lead to partially different impact of tDCS on task-related brain activity and connectivity. Thus, together with previous evidence from single-group resting-state fMRI studies (Antonenko et al., 2017; Lindenberg et al., 2013), Martin et al.'s results suggest an impact of age on tDCS-induced network modulations.

Here, we extend previous work by providing first evidence for an age-dependent effect of anodal stimulation over the sensorimotor cortex on resting-state functional connectivity, indicating differing and potentially opponent modulatory patterns in older and young adults. Brain areas under the target anodal electrode, including left paracentral gyrus, superior parietal cortex and precuneus showed increased centrality in young, but decreased centrality in older adults. This result indicates that brain areas around and posterior to the sensorimotor cortex may become more tightly connected during anodal stimulation in young adults. Thus, their relative role for efficient communication with the rest of the brain is increased. In older adults, on the contrary, the tDCS-induced global connectivity reorganization results in a diminished role of these hubs within the functional network. These findings complement previous suggestions of differential effects of such interventions due to age-associated reorganization of intra- and inter-network connections (Geerligs et al., 2015; Martin et al., 2017; Perceval et al., 2016). In particular, the downregulation or decoupling of involved brain areas may be a core underlying mechanism of anodal stimulation in older adults.

In fact, adjunctive seed-based functional connectivity analyses yielded support for this hypothesis. Using the most significant cluster from centrality analyses, which corresponded to the brain area below the stimulation electrode as a seed, we found differential modulatory 
pattern during anodal compared to sham stimulation in older and young adults. In young adults, the increase in network coupling seems to originate from augmented connectivity with ventromedial prefrontal areas. Alterations in older adults were related to reduced connectivity with posteromedial brain areas. Tentatively, effects in young adults may act upon strengthening the connection between sensorimotor and prefrontal "higher-order" systems. In contrast, in older adults, anodal stimulation effects may originate from effective decoupling between sensorimotor and posterior "lower-order" visual systems. However, the behavioral significance of these distinct neuromodulatory patterns is not clear yet. As we did not acquire behavioral data in our study, we can only speculate, based on previous evidence, that anodal tDCS would potentially cause positive stimulation effects on motor function in both age groups (Perceval et al., 2016; Stagg and Nitsche, 2011), but with varying underlying neural substrates.

Lastly, we want to emphasize that it remains to be clarified whether our results generalize to other electrode configurations, stimulation parameters and study designs, highlighting the importance and non-triviality of replicating and extending our observations while varying methodological factors. In addition, intra- and inter-individually varying factors have recently become the focus of attention - and should be considered in addition to group analyses - in brain stimulation approaches due to their impact on individual responsiveness to tDCS and thus its functional outcome (Bergmann et al., 2016; Berryhill, 2017; Krause and Cohen Kadosh, 2014; Polania et al., 2018; Stephens et al., 2017).

\section{Conclusions}

This is the first systematic investigation of age-related differences in tDCS-induced modulation of resting-state functional network connectivity. We confirm and extend previous evidence for neuromodulatory effects in the aging brain by providing a detailed picture of distinct stimulation-induced global brain network reorganization. Importantly, we observed an interaction between the effect of anodal stimulation and age group, reflecting opposite patterns of network centrality modulation in older and young adults. Increased centrality 
values in young and decreased centrality values in older adults during anodal stimulation likely originated from differential functional coupling of the stimulated brain area. This work contributes to the understanding of complex neural mechanisms underlying brain stimulation.

Future studies should assess the impact of these and other stimulation parameters on various functional networks, and relate neural effects to behavioral outcomes in order to probe the functional significance of differential tDCS-induced neuromodulation between age groups. Further, their temporal dynamics remain to be evaluated in order to reveal whether the effects outlast the stimulation interval.

\section{Acknowledgements}

The authors thank Semiha Aydin, Florian Bohm, and Angelica Sousa for help with data acquisition and Dr. Ulrike Grittner for statistical assistance. This work was supported by the "Bundesministerium für Bildung und Forschung" [01GQ1424A]. Conflicts of interest: none.

\section{References}

Adriaanse, S.M., Wink, A.M., Tijms, B.M., Ossenkoppele, R., Verfaillie, S.C., Lammertsma, A.A., Boellaard, R., Scheltens, P., van Berckel, B.N., Barkhof, F., 2016. The Association of Glucose Metabolism and Eigenvector Centrality in Alzheimer's Disease. Brain Connect 6, 1-8.

Agosta, F., Pievani, M., Geroldi, C., Copetti, M., Frisoni, G.B., Filippi, M., 2012. Resting state fMRI in Alzheimer's disease: beyond the default mode network. Neurobiol Aging 33, 1564-1578.

Antal, A., Polania, R., Schmidt-Samoa, C., Dechent, P., Paulus, W., 2011. Transcranial direct current stimulation over the primary motor cortex during fMRI. Neuroimage 55, 590-596.

Antonenko, D., Kulzow, N., Sousa, A., Prehn, K., Grittner, U., Floel, A., 2018. Neuronal and behavioral effects of multi-day brain stimulation and memory training. Neurobiol Aging 61, 245-254.

Antonenko, D., Schubert, F., Bohm, F., Ittermann, B., Aydin, S., Hayek, D., Grittner, U., Floel, A., 2017. tDCS-Induced Modulation of GABA Levels and Resting-State Functional Connectivity in Older Adults. J Neurosci 37, 4065-4073.

Ashburner, J., 2007. A fast diffeomorphic image registration algorithm. Neuroimage 38, 95-113.

Bachtiar, V., Near, J., Johansen-Berg, H., Stagg, C.J., 2015. Modulation of GABA and resting state functional connectivity by transcranial direct current stimulation. Elife 4, e08789.

Behzadi, Y., Restom, K., Liau, J., Liu, T.T., 2007. A component based noise correction method (CompCor) for BOLD and perfusion based fMRI. Neuroimage 37, 90-101.

Bergmann, T.O., Karabanov, A., Hartwigsen, G., Thielscher, A., Siebner, H.R., 2016. Combining noninvasive transcranial brain stimulation with neuroimaging and electrophysiology: Current approaches and future perspectives. Neuroimage 140, 4-19.

Berryhill, M.E., 2017. Longitudinal tDCS: Consistency across Working Memory Training Studies. AIMS Neuroscience 4, 71-86. 
Binnewijzend, M.A., Adriaanse, S.M., Van der Flier, W.M., Teunissen, C.E., de Munck, J.C., Stam, C.J., Scheltens, P., van Berckel, B.N., Barkhof, F., Wink, A.M., 2014. Brain network alterations in Alzheimer's disease measured by eigenvector centrality in $\mathrm{fMRI}$ are related to cognition and CSF biomarkers. Hum Brain Mapp 35, 2383-2393.

Bolognini, N., Miniussi, C., 2016. Multimodal Association of tDCS with Electroencephalography. In: Brunoni, A., Nitsche, M., Loo, C. (Eds.), Transcranial Direct Current Stimulation in Neuropsychiatric Disorders: Clinical Principles and Management. Springer International Publishing, Cham, pp. 153-168. Bonacich, P., 2007. Some unique properties of eigenvector centrality. Soc Networks 29, 555-564.

Bullmore, E., Sporns, O., 2009. Complex brain networks: graph theoretical analysis of structural and functional systems. Nat Rev Neurosci 10, 186-198.

Bungert, A., Antunes, A., Espenhahn, S., Thielscher, A., 2017. Where does TMS Stimulate the Motor Cortex? Combining Electrophysiological Measurements and Realistic Field Estimates to Reveal the Affected Cortex Position. Cereb Cortex 27, 5083-5094.

Chai, X.J., Castanon, A.N., Ongur, D., Whitfield-Gabrieli, S., 2012. Anticorrelations in resting state networks without global signal regression. Neuroimage 59, 1420-1428.

Datta, A., Bansal, V., Diaz, J., Patel, J., Reato, D., Bikson, M., 2009. Gyri-precise head model of transcranial direct current stimulation: improved spatial focality using a ring electrode versus conventional rectangular pad. Brain Stimul 2, 201-207, 207.e201.

Davis, S.W., Dennis, N.A., Daselaar, S.M., Fleck, M.S., Cabeza, R., 2008. Que PASA? The posterioranterior shift in aging. Cereb Cortex 18, 1201-1209.

Dennis, N.A., Cabeza, R., 2008. Neuroimaging of healthy cognitive aging. The handbook of aging and cognition 3, 1-54.

Eijlers, A.J., Meijer, K.A., Wassenaar, T.M., Steenwijk, M.D., Uitdehaag, B.M., Barkhof, F., Wink, A.M., Geurts, J.J., Schoonheim, M.M., 2017. Increased default-mode network centrality in cognitively impaired multiple sclerosis patients. Neurology 88, 952-960.

Ferreira, L.K., Busatto, G.F., 2013. Resting-state functional connectivity in normal brain aging. Neurosci Biobehav Rev 37, 384-400.

Fertonani, A., Miniussi, C., 2016. Transcranial Electrical Stimulation: What We Know and Do Not Know About Mechanisms. Neuroscientist [Epub ahead of print].

Forman, S.D., Cohen, J.D., Fitzgerald, M., Eddy, W.F., Mintun, M.A., Noll, D.C., 1995. Improved assessment of significant activation in functional magnetic resonance imaging (fMRI): use of a clustersize threshold. Magn Reson Med 33, 636-647.

Fox, M.D., Raichle, M.E., 2007. Spontaneous fluctuations in brain activity observed with functional magnetic resonance imaging. Nat Rev Neurosci 8, 700-711.

Geerligs, L., Renken, R.J., Saliasi, E., Maurits, N.M., Lorist, M.M., 2015. A Brain-Wide Study of AgeRelated Changes in Functional Connectivity. Cereb Cortex 25, 1987-1999.

Gläscher, J., Gitelman, D., 2008. Contrast weights in flexible factorial design with multiple groups of subjects. SPM@ JISCMAIL. AC. UK) Sml, editor, 1-12.

Hartwigsen, G., Bergmann, T.O., Herz, D.M., Angstmann, S., Karabanov, A., Raffin, E., Thielscher, A., Siebner, H.R., 2015. Modeling the effects of noninvasive transcranial brain stimulation at the biophysical, network, and cognitive level. Prog Brain Res 222, 261-287.

Johnson, S.C., Saykin, A.J., Baxter, L.C., Flashman, L.A., Santulli, R.B., McAllister, T.W., Mamourian, A.C., 2000. The relationship between fMRI activation and cerebral atrophy: comparison of normal aging and alzheimer disease. Neuroimage 11, 179-187.

Johnstone, A., Hinson, E., Stagg, C.J., 2016. tDCS and Magnetic Resonance Imaging. In: Brunoni, A., Nitsche, M., Loo, C. (Eds.), Transcranial Direct Current Stimulation in Neuropsychiatric Disorders: Clinical Principles and Management. Springer International Publishing, Cham, pp. 169-195.

Jones, D.T., Machulda, M.M., Vemuri, P., McDade, E.M., Zeng, G., Senjem, M.L., Gunter, J.L., Przybelski, S.A., Avula, R.T., Knopman, D.S., Boeve, B.F., Petersen, R.C., Jack, C.R., Jr., 2011. Agerelated changes in the default mode network are more advanced in Alzheimer disease. Neurology 77 , 1524-1531.

Jones, K.T., Gozenman, F., Berryhill, M.E., 2015. The strategy and motivational influences on the beneficial effect of neurostimulation: a tDCS and fNIRS study. Neuroimage 105, 238-247.

Kim, J.H., Kim, D.W., Chang, W.H., Kim, Y.H., Kim, K., Im, C.H., 2014. Inconsistent outcomes of transcranial direct current stimulation may originate from anatomical differences among individuals: electric field simulation using individual MRI data. Neurosci Lett 564, 6-10.

Krause, B., Cohen Kadosh, R., 2014. Not all brains are created equal: the relevance of individual differences in responsiveness to transcranial electrical stimulation. Front Syst Neurosci 8, 25.

Kuo, M.F., Nitsche, M.A., 2012. Effects of transcranial electrical stimulation on cognition. Clin EEG Neurosci 43, 192-199. 
Laakso, I., Tanaka, S., Koyama, S., De Santis, V., Hirata, A., 2015. Inter-subject Variability in Electric Fields of Motor Cortical tDCS. Brain Stimul 8, 906-913.

Lindenberg, R., Nachtigall, L., Meinzer, M., Sieg, M.M., Floel, A., 2013. Differential effects of dual and unihemispheric motor cortex stimulation in older adults. J Neurosci 33, 9176-9183.

Lindenberg, R., Sieg, M.M., Meinzer, M., Nachtigall, L., Floel, A., 2016. Neural correlates of unihemispheric and bihemispheric motor cortex stimulation in healthy young adults. Neuroimage 140, 141-149.

Lohmann, G., Margulies, D.S., Horstmann, A., Pleger, B., Lepsien, J., Goldhahn, D., Schloegl, H., Stumvoll, M., Villringer, A., Turner, R., 2010. Eigenvector centrality mapping for analyzing connectivity patterns in fMRI data of the human brain. PLoS One 5, e10232.

Long, X., Huang, W., Napadow, V., Liang, F., Pleger, B., Villringer, A., Witt, C.M., Nierhaus, T., Pach, D., 2016. Sustained Effects of Acupuncture Stimulation Investigated with Centrality Mapping Analysis. Front Hum Neurosci 10, 510.

Luo, X., Qiu, T., Jia, Y., Huang, P., Xu, X., Yu, X., Shen, Z., Jiaerken, Y., Guan, X., Zhou, J., Zhang, M., Adni, 2016. Intrinsic functional connectivity alterations in cognitively intact elderly APOE epsilon4 carriers measured by eigenvector centrality mapping are related to cognition and CSF biomarkers: a preliminary study. Brain Imaging Behav 11, 1290-1301.

Mahdavi, S., Towhidkhah, F., Alzheimer's Disease Neuroimaging, I., 2018. Computational human head models of tDCS: Influence of brain atrophy on current density distribution. Brain Stimul 11, 104107.

Mameli, F., Fumagalli, M., Ferrucci, R., Priori, A., 2014. Transcranial Direct Current Stimulation and Cognition in the Elderly. In: Kadosh, R.C. (Ed.), The Stimulated Brain. Elsevier, London, pp. 371-395. Marangolo, P., Fiori, V., Sabatini, U., De Pasquale, G., Razzano, C., Caltagirone, C., Gili, T., 2016. Bilateral Transcranial Direct Current Stimulation Language Treatment Enhances Functional Connectivity in the Left Hemisphere: Preliminary Data from Aphasia. J Cogn Neurosci 28, 724-738.

Martin, A., Meinzer, M., Lindenberg, R., Sieg, M.M., Nachtigall, L., Floel, A., 2017. Effects of Transcranial Direct Current Stimulation on Neural Network Structure in Young and Older Adults. J Cogn Neurosci, 1-12.

Meinzer, M., Antonenko, D., Lindenberg, R., Hetzer, S., Ulm, L., Avirame, K., Flaisch, T., Floel, A., 2012. Electrical brain stimulation improves cognitive performance by modulating functional connectivity and task-specific activation. J Neurosci 32, 1859-1866.

Meinzer, M., Lindenberg, R., Darkow, R., Ulm, L., Copland, D., Floel, A., 2014. Transcranial direct current stimulation and simultaneous functional magnetic resonance imaging. $J$ Vis Exp.

Meinzer, M., Lindenberg, R., Phan, M.T., Ulm, L., Volk, C., Floel, A., 2015. Transcranial direct current stimulation in mild cognitive impairment: Behavioral effects and neural mechanisms. Alzheimers Dement 11, 1032-1040.

Miniussi, C., Brignani, D., Pellicciari, M.C., 2012. Combining transcranial electrical stimulation with electroencephalography: a multimodal approach. Clin EEG Neurosci 43, 184-191.

Monte-Silva, K., Kuo, M.F., Hessenthaler, S., Fresnoza, S., Liebetanz, D., Paulus, W., Nitsche, M.A., 2013. Induction of late LTP-like plasticity in the human motor cortex by repeated non-invasive brain stimulation. Brain Stimul 6, 424-432.

Nierhaus, T., Forschack, N., Piper, S.K., Holtze, S., Krause, T., Taskin, B., Long, X., Stelzer, J., Margulies, D.S., Steinbrink, J., Villringer, A., 2015. Imperceptible somatosensory stimulation alters sensorimotor background rhythm and connectivity. J Neurosci 35, 5917-5925.

Nierhaus, T., Margulies, D.S., Long, X., Villringer, A., 2012. fMRI for the assessment of functional connectivity. In: Bright, P. (Ed.), Neuroimaging methods. In Tech, Rijeka.

Nitsche, M.A., Paulus, W., 2000. Excitability changes induced in the human motor cortex by weak transcranial direct current stimulation. J Physiol 527 Pt 3, 633-639.

Nitsche, M.A., Paulus, W., 2001. Sustained excitability elevations induced by transcranial DC motor cortex stimulation in humans. Neurology 57, 1899-1901.

Nitsche, M.A., Polania, R., Kuo, M.-F., 2015. Transcranial Direct Current Stimulation: Modulation of Brain Pathways and Potential Clinical Applications. Brain Stimulation. John Wiley \& Sons, Ltd, pp. 233-254.

Opitz, A., Paulus, W., Will, S., Antunes, A., Thielscher, A., 2015. Determinants of the electric field during transcranial direct current stimulation. Neuroimage 109, 140-150.

Perceval, G., Floel, A., Meinzer, M., 2016. Can transcranial direct current stimulation counteract ageassociated functional impairment? Neurosci Biobehav Rev 65, 157-172.

Polania, R., Nitsche, M.A., Ruff, C.C., 2018. Studying and modifying brain function with non-invasive brain stimulation. Nat Neurosci. 
Polanía, R., Paulus, W., Antal, A., Nitsche, M.A., 2011. Introducing graph theory to track for neuroplastic alterations in the resting human brain: A transcranial direct current stimulation study. Neuroimage 54, 2287-2296.

Poreisz, C., Boros, K., Antal, A., Paulus, W., 2007. Safety aspects of transcranial direct current stimulation concerning healthy subjects and patients. Brain Res Bull 72, 208-214.

Sala-Llonch, R., Bartres-Faz, D., Junque, C., 2015. Reorganization of brain networks in aging: a review of functional connectivity studies. Front Psychol 6, 663.

Saturnino, G.B., Antunes, A., Thielscher, A., 2015. On the importance of electrode parameters for shaping electric field patterns generated by tDCS. Neuroimage 120, 25-35.

Sehm, B., Schafer, A., Kipping, J., Margulies, D., Conde, V., Taubert, M., Villringer, A., Ragert, P., 2012. Dynamic modulation of intrinsic functional connectivity by transcranial direct current stimulation. J Neurophysiol 108, 3253-3263.

Siebner, H.R., Bergmann, T.O., Bestmann, S., Massimini, M., Johansen-Berg, H., Mochizuki, H., Bohning, D.E., Boorman, E.D., Groppa, S., Miniussi, C., Pascual-Leone, A., Huber, R., Taylor, P.C., Ilmoniemi, R.J., De Gennaro, L., Strafella, A.P., Kahkonen, S., Kloppel, S., Frisoni, G.B., George, M.S., Hallett, M., Brandt, S.A., Rushworth, M.F., Ziemann, U., Rothwell, J.C., Ward, N., Cohen, L.G., Baudewig, J., Paus, T., Ugawa, Y., Rossini, P.M., 2009. Consensus paper: combining transcranial stimulation with neuroimaging. Brain Stimul 2, 58-80.

Stagg, C.J., Nitsche, M.A., 2011. Physiological basis of transcranial direct current stimulation. Neuroscientist 17, 37-53.

Stephens, J.A., Jones, K.T., Berryhill, M.E., 2017. Task demands, tDCS intensity, and the COMT val(158)met polymorphism impact tDCS-linked working memory training gains. Sci Rep 7, 13463.

Summers, J.J., Kang, N., Cauraugh, J.H., 2016. Does transcranial direct current stimulation enhance cognitive and motor functions in the ageing brain? A systematic review and meta- analysis. Ageing Res Rev 25, 42-54.

Taubert, M., Lohmann, G., Margulies, D.S., Villringer, A., Ragert, P., 2011. Long-term effects of motor training on resting-state networks and underlying brain structure. Neuroimage 57, 1492-1498.

Thielscher, A., Antunes, A., Saturnino, G.B., 2015. Field modeling for transcranial magnetic stimulation: A useful tool to understand the physiological effects of TMS? Conf Proc IEEE Eng Med Biol Soc 2015, 222-225.

Thomas, C., Datta, A., Woods, A., 2017. Effect of aging on current flow due to transcranial direct current stimulation. Brain Stimulation: Basic, Translational, and Clinical Research in Neuromodulation $10,469$.

van Duinkerken, E., Schoonheim, M.M., RG, I.J., Moll, A.C., Landeira-Fernandez, J., Klein, M., Diamant, M., Snoek, F.J., Barkhof, F., Wink, A.M., 2017. Altered eigenvector centrality is related to local resting-state network functional connectivity in patients with longstanding type 1 diabetes mellitus. Hum Brain Mapp [Epub ahead of print].

Watson, C., Clark, C.M., Tellegen, A., 1988. Development and validation of brief measures of positive and negative affect: the PANAS scales. J Pers Soc Psychol 54, 1063-1070.

Wiepert, D.A., Lowe, V.J., Knopman, D.S., Boeve, B.F., Graff-Radford, J., Petersen, R.C., Jack, C.R., Jr., Jones, D.T., 2017. A robust biomarker of large-scale network failure in Alzheimer's disease. Alzheimers Dement (Amst) 6, 152-161.

Windhoff, M., Opitz, A., Thielscher, A., 2013. Electric field calculations in brain stimulation based on finite elements: an optimized processing pipeline for the generation and usage of accurate individual head models. Hum Brain Mapp 34, 923-935.

Wink, A.M., de Munck, J.C., van der Werf, Y.D., van den Heuvel, O.A., Barkhof, F., 2012. Fast eigenvector centrality mapping of voxel-wise connectivity in functional magnetic resonance imaging: implementation, validation, and interpretation. Brain Connect 2, 265-274.

Woods, A.J., Antal, A., Bikson, M., Boggio, P.S., Brunoni, A.R., Celnik, P., Cohen, L.G., Fregni, F., Herrmann, C.S., Kappenman, E.S., Knotkova, H., Liebetanz, D., Miniussi, C., Miranda, P.C., Paulus, W., Priori, A., Reato, D., Stagg, C., Wenderoth, N., Nitsche, M.A., 2016. A technical guide to tDCS, and related non-invasive brain stimulation tools. Clin Neurophysiol 127, 1031-1048.

Zhang, H., Lee, A., Qu, A., 2017. A posterior-to-anterior shift of brain functional dynamics in aging. Brain Struct Funct 222, 3665-3676.

Zuo, X.N., Ehmke, R., Mennes, M., Imperati, D., Castellanos, F.X., Sporns, O., Milham, M.P., 2012. Network centrality in the human functional connectome. Cereb Cortex 22, 1862-1875. 